\title{
Tissue Aortic Valve
}

National Cancer Institute

\section{Source}

National Cancer Institute. Tissue Aortic Valve. NCI Thesaurus. Code C80469.

A valve made of animal tissues or harvested from human cadavers and used to replace the aortic valve in patients with aortic valve stenosis or regurg itation. 